\title{
Cellular Neural Networks for NP-Hard Optimization
}

\author{
Mária Ercsey-Ravasz, ${ }^{1}$ Tamás Roska, ${ }^{2,3}$ and Zoltán Néda ${ }^{4}$ \\ ${ }^{1}$ Department of Physics, University of Notre Dame, Notre Dame, IN 46556, USA \\ ${ }^{2}$ Faculty of Information Technology, Péter Pázmany Catholic University, 1083 Budapest, Hungary \\ ${ }^{3}$ Computer and Automation Research Institute, Hungarian Academy of Sciences (MTA-SZTAKI), 1111 Budapest, Hungary \\ ${ }^{4}$ Faculty of Physics, Babeş-Bolyai University, 400084 Cluj-Napoca, Romania
}

Correspondence should be addressed to Mária Ercsey-Ravasz, mercseyr@nd.edu

Received 24 September 2008; Accepted 26 November 2008

Recommended by David Lopez Vilarino

A cellular neural/nonlinear network $(\mathrm{CNN})$ is used for NP-hard optimization. We prove that a CNN in which the parameters of all cells can be separately controlled is the analog correspondent of a two-dimensional Ising-type (Edwards-Anderson) spin-glass system. Using the properties of CNN, we show that one single operation (template) always yields a local minimum of the spin-glass energy function. This way, a very fast optimization method, similar to simulated annealing, can be built. Estimating the simulation time needed on CNN-based computers, and comparing it with the time needed on normal digital computers using the simulated annealing algorithm, the results are astonishing. CNN computers could be faster than digital computers already at $10 \times 10$ lattice sizes. The local control of the template parameters was already partially realized on some of the hardwares, we think this study could further motivate their development in this direction.

Copyright (C) 2009 Mária Ercsey-Ravasz et al. This is an open access article distributed under the Creative Commons Attribution License, which permits unrestricted use, distribution, and reproduction in any medium, provided the original work is properly cited.

\section{Introduction}

NP-hard optimization problems represent a key task when testing novel computing paradigms. These complex problems frequently appear in physics, life sciences, biometrics, logistics, database search, and so on, and in most cases they are associated with important practical applications as well [1]. The deficiency of solving these problems in a reasonable amount of time is one of the most important limitation of digital computers, thus all novel paradigms are tested in this sense. Quantum computing, for example, is theoretically well suited for solving NP-hard problems, but the technical realization of quantum computers seems to be quite hard. Here, we argue that cellular neural networks (CNNs) show good perspectives for fast NP-hard optimization. The advantage of cellular wave computing [2], and the CNN paradigm [3], relative to quantum computing is that several practical realizations are already available. After the idea of CNN appeared in 1988 [4], a detailed plan for a CNN computer was developed in 1993 [5]. Since then the chips had a fast developing trend $[6,7]$ and the latest-already commercialized-version is the Q-Eye chip with lattice size $176 \times 144$, included in the Eye-RIS system, mainly used as a visual microprocessor [8].

It has been proved in several previous studies that this novel computational paradigm is useful in solving partial differential equations $[9,10]$, studying cellular automata models $[11,12]$, doing image processing [13], and making Monte Carlo simulations on lattice models [14, 15]. Here, it is shown that NP-hard optimization algorithms could be also highly effective using the CNN-UM, if the parameters (templates) of each cell could be separately controlled. The local control of the parameters of each cell is already partially realized on some hardwares and the importance of this study consists also in motivating the further development of hardwares in such direction. In Section 2, we will shortly present the chosen NP-hard problem, namely, the optimization of spin-glass systems. After that, in Section 3, we prove that a CNN computer, on which the parameters of each cell can be separately controlled, is the analog correspondent of a locally coupled two-dimensional spin-glass system. Using these properties of CNN computers, a fast optimization algorithm can be thus 
developed, presented in Section 4. In Section 5, we make a rough estimation of the speed of the algorithm, based on the properties of existing hardwares.

\section{Spin-Glass Systems}

The NP-hard problem we chose to solve using a locally variant $\mathrm{CNN}$ is a two-dimensional Ising-type (EdwardsAnderson) spin-glass system [16, 17]. A spin glass is a disordered material exhibiting high magnetic frustration. The typical origin of this frustration is the simultaneous presence of competing interactions and disorder. The Edwards-Anderson model places the interacting Ising-type spins (which can have two possible states $\sigma_{i, j}= \pm 1$ ) on a square lattice. They interact through an exchange interaction with their neighbors. The strength of the interaction is characterized by the $J_{i, j ; k, l}$ coupling constants, separately defined for each connection. The typical frustration characteristic for spin glasses appears, when the coupling constants can take both positive (favoring the spins to align in the same direction) and negative values (favoring the spins to align in opposite directions).

The energy function of the system is the following:

$$
H=-\sum_{\langle i, j ; k, l\rangle} J_{i, j ; k, l} \sigma_{i, j} \sigma_{k, l}
$$

$\langle i, j ; k, l\rangle$ representing neighbors, $J_{i, j ; k, l}$ is the coupling constant between the spins with coordinates $(i, j)$ and $(k, l)$. If external magnetic field is also added, then the energy is

$$
H=-\sum_{\langle i, j ; k, l\rangle} J_{i, j ; k, l} \sigma_{i, j} \sigma_{k, l}-\sum_{i, j} B_{i, j} \sigma_{i, j}
$$

where $B_{i, j}$ is the strength of the external magnetic field at the lattice site with coordinates $(i, j)$.

The energy landscape of spin-glasses is very complicated, there are many local minima, and finding the ground state is in most of the cases an NP-hard problem. The exceptions are the systems defined on planar graphs, for example, the two-dimensional lattice on which spins are only connected to their 4 nearest neighbors. This can be solved in polynomial time [18]. However, any two-dimensional graph or lattice structure, in which crossing bonds exist, is a nonplanar graph and the problem is already NP-hard (for details see [19]). In this paper, we concentrate on the two dimensional case in which connections with 8 neighbors (nearest and nextnearest neighbors) exist.

Besides its importance in condensed matter physics, spin-glass theory has in the time acquired a strongly interdisciplinary character, with applications to neural network theory [20], computer science [1], theoretical biology [21], econophysics [22], and so on. It has also been shown that using spin-glass models as error-correcting codes, their costperformance is excellent [23].

\section{The Analogy between $\mathrm{CNN}$ and Spin-Glass Systems}

Here, we present a locally variant $\mathrm{CNN}$, which is the analog correspondent of a spin-glass type system. All the local energy minimums of the two systems coincide, the only difference is that in CNN the state variables of the cells are continuous values between -1 and 1 , not discrete values, \pm 1 , like in the Ising-type spin systems.

We consider a two-dimensional CNN, where the templates (parameters of the state equation in the ChuaYang model [4]) can be locally controlled. The feedback matrix, $A$, is considered symmetric $A(i, j ; k, l)=A(k, l ; i, j)$, $A(i, j ; i, j)=1$ for all $(i, j)$, and the elements are bounded $A(i, j ; k, l) \in[-1,1]((i, j)$ and $(k, l)$ denote two neighboring cells). Matrix $B$, which controls the effect of the input array, will be taken simply as $B(i, j ; i, j)=b$ and $B(i, j ; k, l)=0$, $\{i, j\} \neq\{k, l\}$. The parameter $z$ is chosen as $z=0$, so finally our template is defined by $\{A, b\}$ alone. The state-equation of the system writes as

$$
\frac{d x_{i, j}(t)}{d t}=-x_{i, j}(t)+\sum_{\langle k, l\rangle \in N(i, j)} A_{i, j ; k, l} y_{k, l}(t)+b u_{i, j},
$$

where $x_{i, j}$ is the state value, $y_{i, j}$ is the output, and $u_{i, j}$ is the input of the cell $(i, j)$ with neighborhood $N(i, j)$ (8 neighbors and itself).

In their work Chua and Yang [4] defined the Lyapunov function for the CNN, which behaves like the "energy" (Hamiltonian) of the system. For the standard CNN model, this function was defined as follows:

$$
\begin{aligned}
E(t)= & -\frac{1}{2} \sum_{(i, j)} \sum_{(k, l)} A_{i, j ; k, l} y_{i, j}(t) y_{k, l}(t)+\frac{1}{2} \sum_{(i, j)} y_{i, j}(t)^{2} \\
& -\sum_{(i, j)} \sum_{(k, l)} B_{i, j ; k, l} y_{i, j}(t) u_{k, l}-\sum_{(i, j)} z_{i, j} y_{i, j}(t) .
\end{aligned}
$$

Inserting the template parameters defined above, this function can be written simply as

$$
E(t)=-\sum_{\langle i, j ; k, l\rangle} A_{i, j ; k, l} y_{i, j} y_{k, l}-b \sum_{i, j} y_{i, j} u_{i, j} .
$$

Note that taking all $A_{i, j ; i, j}=1$ the second term of (4) disappears, and in $(5)\langle i, j ; k, l\rangle$ denotes pairs of neighbors $((i, j) \neq(k, l))$, each pair taken only once in the sum. $y_{i, j}$ denotes the output value of each cell and $u_{i, j}$ stands for an arbitrary input image.

By choosing the parameter $b=0$, the energy of this special CNN is similar with the energy of the spin-glass system shown in (1). The difference is that Ising spins are defined as \pm 1 , while here we have continuous values between $[-1,1]$. When $b \neq 0$ the Lyapunov function has a similar form to (2), the input image takes the role of the external magnetic field, and parameter $b$ can globally influence the strength of this external field.

The $A(i, j ; k, l)$ parameters take the role of the $J_{i, j ; k, l}$ coupling constants, and they can be positive and negative as well. In the following, we will be especially interested in the 
case when the $A(i, j ; k, l)$ couplings lead to a frustration and the quenched disorder in the system is similar with that of spin-glass systems $[16,17]$.

For showing the analogy between this $\mathrm{CNN}$ and spinglass system, we will use three important properties of the $\mathrm{CNN}$. The first two concerns the Lyapunov function defined by Chua and Yang [4].

(1) It is always a monotone decreasing function in time, $d E / d t \leq 0$, so starting from an initial condition $E$ can only decrease during the dynamics of the CNN.

(2) The final steady state is a local minimum of the energy $d E / d t=0$.

We shortly prove this theorem for the energy function defined in our system. Making the derivative of the energy function, one gets

$$
\begin{aligned}
\frac{d E}{d t}= & -\sum_{\langle i, j ; k, l\rangle} A_{i, j ; k, l}\left(\frac{d y_{i, j}}{d x_{i, j}} \frac{d x_{i, j}}{d t} y_{k, l}+y_{i, j} \frac{d y_{k, l}}{d x_{k, l}} \frac{d x_{k, l}}{d t}\right) \\
& -b \sum_{i, j} \frac{d y_{i, j}}{d x_{i, j}} \frac{d x_{i, j}}{d t} u_{i, j} \\
= & -\sum_{i, j} \sum_{k, l} \frac{d y_{i, j}}{d x_{i, j}} \frac{d x_{i, j}}{d t} A_{i, j ; k, l} y_{k, l}-b \sum_{i, j} \frac{d y_{i, j}}{d x_{i, j}} \frac{d x_{i, j}}{d t} u_{i, j} \\
= & -\sum_{i, j} \sum_{k, l} \frac{d y_{i, j}}{d x_{i, j}} \frac{d x_{i, j}}{d t}\left(A_{i, j ; k, l} y_{k, l}+b * u_{i, j}\right) \\
= & -\sum_{i, j} \sum_{k, l} \frac{d y_{i, j}}{d x_{i, j}}\left(\frac{d x_{i, j}}{d t}\right)^{2} \leq 0 .
\end{aligned}
$$

Note that the symmetry property of the $A$ template was used $A(i, j ; k, l)=A(k, l ; i, j)$. From the properties of the output function defined as $y_{i, j}=(1 / 2)\left(\left|x_{i, j}+1\right|-\left|x_{i, j}-1\right|\right)$ in the Chua-Yang model [4], we know that

$$
\frac{d y_{i, j}}{d x_{i, j}} \geq 1
$$

This way follows that $d E / d t \leq 0$ and in the final steady state when $d x_{i, j} / d t=0$, the energy also reaches a minimum $d E / d t=0$.

In addition to these, our CNN has also another important property. Due to the fact that $A$ is symmetric and all self-interaction parameters are $A(i, j ; i, j)=1$, the output values of the cells in a final steady state will be either 1 or -1 , so the local minima achieved by the CNN is an Isinglike configuration. This can be understood by analyzing the driving-point (DP) plot of the system. The derivative of the state value is plotted in function of the state value in Figure 1. The state equation of a cell can be divided in two parts, one depending on the state value of the cell, $g\left(x_{i, j}(t)\right)$, and the other part depending only on the neighbors:

$$
\frac{d x_{i, j}(t)}{d t}=g\left(x_{i, j}(t)\right)+w(t)
$$

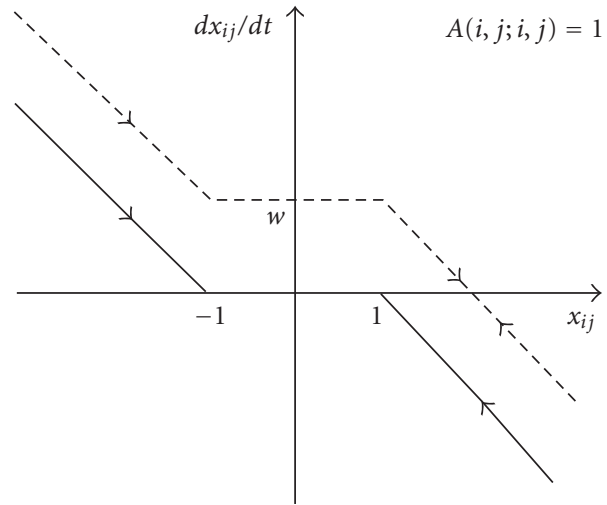

FIgure 1: The DP plot of a cell. The derivative of the state value is presented in function of the state value, for $w(t)=0$ (continuous line) and $w(t)>0$ (dashed line).

where

$$
\begin{aligned}
g\left(x_{i, j}(t)\right) & =-x_{i, j}(t)+A(i, j ; i, j) * f\left(x_{i, j}(t)\right), \\
w(t) & =\sum_{\langle k, l\rangle \neq\langle i, j\rangle} A_{i, j ; k, l} y_{k, l}(t)+b u_{i, j} .
\end{aligned}
$$

Because we have a coupled template in which $w(t)$ changes in time, also the DP plot of the system changes in function of $w(t)$ (in Figure 1 the case of $w(t)=0$ is plotted with a continuous line, and $w(t)>0$ is represented with dashed line). We cannot predict the exact final steady state of the cell, but we can see that the equilibrium points cannot be in the $(-1,1)$ region, so the output of the cell will be always \pm 1 . The single exceptions will be the cells (spins) with 0 local energy, in which case $w(t)=0$. It can happen that these cells do not achieve a \pm 1 output (although on real hardwares, in presence of real noise this is hardly probable), but the state of these does not affect the energy of the Ising configuration. So we can just randomly choose between - land 1 . The result given by the $\mathrm{CNN}$ is similar to finding more than one state at the same time.

We can conclude thus, that starting from any initial condition, the final steady state of the CNN template will be always a local minimum of the spin-glass type Ising spin system with local connections defined by matrix $A$. The fact that one single operation is needed for finding a local minimum of the energy gives us hope to develop fast optimization algorithms.

\section{The Optimization Algorithm}

As already emphasized, the complex frustrated case (locally coupled spin-glass-type system), where the $A$ coupling parameters generate a nontrivial quenched disorder will be considered here. The minimum energy configuration of such systems is searched by an algorithm which is similar to the well-known simulated annealing method [24].

The noise is included with random input images $\left(u_{i, j}\right.$ values in (5)) acting as an external locally variable magnetic field. The strength of this field is governed through parameter 


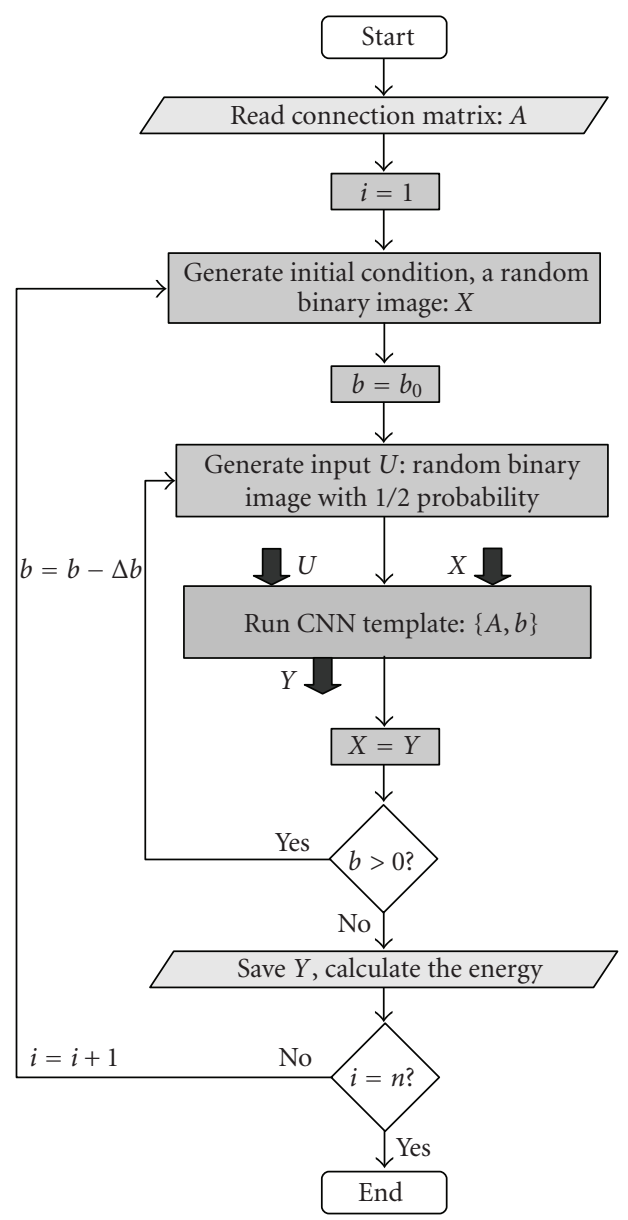

FIGURE 2: Flowchart of the optimization algorithm using $n$ number of cooling processes.

$b$. Whenever $b$ is different from zero, our CNN template minimizes the energy with form (5). The first part of it being the energy of the considered spin-glass-type model and the second part an additional term, which gets minimal when the state of the system is equal to the input image (the external magnetic field). If $b$ is large, the result will be the input image itself, if $b=0$ the result is a local minimum of the pure Ising-type system. For values in between, our result is a "compromise" between the two cases. Slowly decreasing the value of $b$ will result in a process similar with simulated annealing, where the temperature of the system is consecutively lowered. First, big fluctuations of the energy are allowed, and by decreasing this we slowly drive the system to a low-energy state. Since the method is a stochastic one, we can, of course, never be totally sure that the global minimum will be achieved, but good approximations can be achieved.

The algorithm is as follows (see Figure 2).

(1) One starts from a random initial condition $x$, and $b=$ 5 (with this value the result of the template is almost exactly the same as the input image).

(2) A binary random input image $u$ is generated with $1 / 2$ probability of black (1) pixels.
(3) Using the $x$ initial state and the $u$ input image, the CNN template is applied.

(4) The value of $b$ is decreased with steps $\Delta b$.

(5) Steps 2-4 are repeated until $b=0$ is reached. The results of the previous step (minimization) are considered always as the initial state for the next step.

(6) When reaching $b=0$, the image (Ising spin configuration) is saved and the energy is calculated.

In the classical-simulated annealing algorithm, several thousands of steps for a single temperature are needed. Here, at each noise value one single CNN template is applied. The settling time of the template may vary (it is usually longer in the beginning and gets very fast at the end of the algorithm), but given the fact that after each step noise is introduced, it is acceptable to set a constant running time for our template (usually 5 times the time constant of the CNN dynamics).

Similarly, with choosing the cooling rate in simulated annealing, choosing the value of $\Delta b$ is also a delicate problem. A proper value providing an acceptable compromise between the quality of the results and speed of the algorithm has to be found. For each system size, one can find an optimal value of $\Delta b$, but as one would expect this is rapidly decreasing by increasing the system size. It is much more effective, both for performance (meaning the probability of finding the real global optimum) and speed to choose a constant $\Delta b=0.05$ step and repeat the whole cooling process several times. As a result, several different final states will be obtained, and we have a higher probability to get the right global minima between these. On the flowchart, the number of cooling processes is denoted with $n$ (Figure 2 ).

For testing the efficiency of the algorithm, one needs to measure the number of steps necessary for finding the right global minima. To do this, one has to previously know the global minima. In case of small systems with $L=5,6$ this can be obtained by a quick exhaustive search in the phase space. For bigger systems, the classical-simulated annealing algorithm was used. The temperature was decreased with a rate of $0.99\left(T_{\text {final }} / T_{\text {ini }}\right)$ and 1000 Monte Carlo steps were performed for each temperature.

In the present work, spin-glass systems with $A(i, j ; k, l)=$ \pm 1 , local connections were studied. The $P$ probability of the positive bonds was varied (influencing the amount of frustration in the system), and local interactions with the 8 Moore neighbors were considered. For several $P$ densities of the positive links and various system sizes, we calculated the average number of steps needed for finding the energy minimum. As naturally is expected for the nontrivial frustrated cases, the number of simulation steps needed exponentially increases with the system size. As an example, the $P=.4$ case is shown in Figure 3(a). As observable in the figure, we could made estimates for relatively small system sizes only $(L<12)$. The reason for this is that we had to simulate also the CNN operations, and for large lattices a huge system of partial differential equations had to be solved. This process gets quite slow for bigger lattices.

The needed average number of steps to reach the estimated energy minima depends also on the $P$ probability 


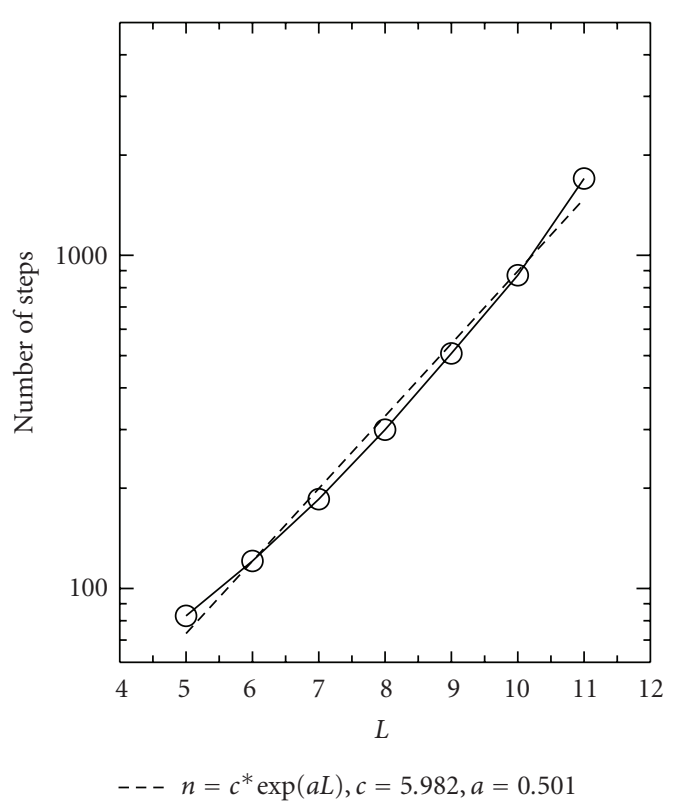

(a)

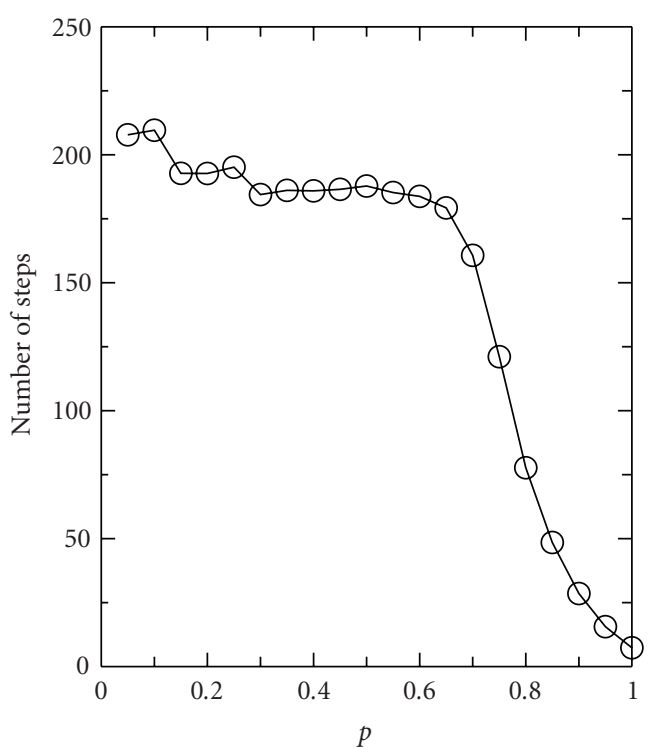

(b)

Figure 3: (a) The number of steps needed to find the optimal energy as a function of the lattice size $L$. The density of positive connections is fixed to $P=.4$, and the parameter $\Delta b=0.05$ is used. (b) For a system with size $L=7$, the number of steps needed for getting the presumed global minima is plotted as a function of the probability of positive connections $p$.

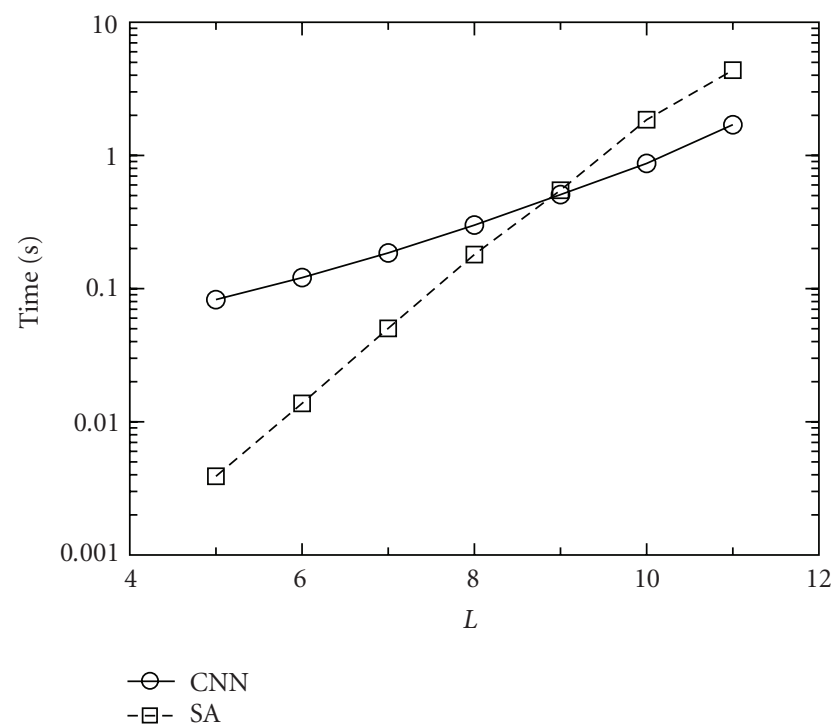

Figure 4: (a) Time needed to reach the minimum energy as a function of the lattice size $L$. Circles are for estimates on CNN computers and squares are simulated annealing results on $3.4 \mathrm{GHz}$ Pentium 4. Results are averaged on 10000 different configurations with $P=.4$ probability of positive bonds. For the $\mathrm{CNN}$ algorithm, $\Delta b=0.05$ was chosen. For simulated annealing, the initial temperature was $T_{0}=0.9$, final temperature $T_{f}=0.2$, and the decreasing rate of the temperature was fixed as 0.99 .

of the positive connections in the system. In Figure 3(b), we illustrate this for a system with size $L=7$. To obtain this data for each $P$-value, 5000 different systems were analyzed. As observable in Figure 3(b), the system is almost equally hard to solve for all $P$-values in the rage of $P \in(0, .6)$.

On real hardwares, one has to think about the fact that noise appears also in the template values, and it may introduce small asymmetries $(A(i, j ; k, l)=A(k, l ; i, j)$ will be only approximative). We also tested this case in our simulations. For $P=.4$ density of the positive connections and for $7 \times 7$ lattice size, 1000 systems were analyzed, introducing a relatively large $10 \%$ noise on the template values. Results show no significant difference, the average value of the number of steps needed was 264.0, while in the symmetric case, for the same system size this average was 265.8. Given the fact that our algorithm is similar to simulated annealing, and noise is introduced in the system at each step, these small asymmetries may only increase the noise, but have no significant effect.

\section{Speed Estimation}

Finally, let us have some thoughts about the estimated speed of such an optimization algorithm. On the CNN chips nowadays available, only parameter $z$ can be locally varied, parameters $A$ and $B$ are $3 \times 3$ matrices, uniformly applied for all cells [6]. The reason for no local control of $A$ and $B$ seems to be more due to the lack of motivations. It is technically possible, of course, that the control unit and template memories of the CNN-UM would be more complicated. This modification would not change too much the properties of the hardwares. Introducing the connection parameters (the template) in the local memories of the chip would take a longer time, but in the specific problem considered here the 
connection parameters have to be introduced only once for each problem, so this would not effect in a detectable manner the speed of calculations.

Based on our previous experience with the ACE16K chip (with sizes $128 \times 128$ ) $[14,15]$, we can make a rough estimation of the speed for the presented optimization algorithm. This chip with its parallel architecture solves one template in a very short time-of the order of microseconds. For each step in the algorithm, one also needs to generate a random binary image. This process is already 4 times faster on the ACE16K chip than on a $3.4 \mathrm{GHz}$ Pentium 4 computer and needs around 100 microseconds (see [14]). It is also helpful for the speed that in the present algorithm it is not needed to save information at each step, only once at the end of each cooling process. Saving an image takes roughly 1 millisecond on the ACE16K, but this is done only once after several thousands of simulation steps. Making thus a rough estimate for our algorithm, a chip with similar properties like the ACE16K should be able to compute between 1000-5000 steps in one second, independently of the lattice size. Using the lower estimation value (1000 steps /second) and following up the number of steps needed in case of $P=.4$, the estimated average time for solving one problem is plotted as a function of the lattice size in Figure 4 (circles).

Comparing this with the speed of simulated annealing (SA) performed on a $3.4 \mathrm{GHz}$ Pentium 4 (squares in Figure 4), the results for larger lattice sizes are clearly in favor of the CNN chips. For testing the speed of simulated annealing, we used the following parameters: initial temperature $T_{0}=0.9$, final temperature $T_{f}=0.2$, decreasing rate of the temperature 0.99. Results were averaged for 10000 different bond distributions. The necessary number of Monte Carlo steps was always carefully measured by performing many different simulations, using different number of Monte Carlo steps, and comparing the obtained results. From Figure 4, it results that the estimated time needed for the presented algorithm on a CNN chip would be smaller than simulated annealing already at $10 \times 10$ lattice sizes.

Spin-glass-like systems have many applications in which global minimum is not crucial to be exactly found, the minimization is needed only with a margin of error. In such cases, the number of requested steps will decrease drastically. As an example in such sense, it has been shown that using spin-glass models as error-correcting codes, their cost performance is excellent [23], and usually the system is not even in the spin-glass phase. In this manner, by using the CNN chip, finding acceptable results could be very fast, even on big lattices.

\section{Conclusion}

A cellular neural network with locally variable parameters was used for finding the optimal state of locally coupled, twodimensional, Ising-type spin-glass systems. By simulating the proposed optimization algorithm for a CNN chip, where all connections can be locally controlled, very good perspectives for solving such NP hard problems were predicted. CNN computers could be faster than digital computers already at a $10 \times 10$ lattice size. Chips with 2 layers of cells were also produced (CACE1k [25]) and increasing the number of layers is expected in the near future. This way, achieving local control could further extend the number of possible applications. On two layers, it is possible to map already a spin system with any connection matrix (even globally coupled spins) and similar stochastic optimization algorithms could be developed, and also other important NP-hard problems (e.g., K-SAT) may become treatable.

\section{Acknowledgments}

This work is supported by a Hungarian ONR Grant no. (N00014-07-1-0350) and a Romanian Consiliul National al Cercetarii Stiintifice din Invatamantul Superior (CNCSIS) no.1571 research Grant (Contract 84/2007).

\section{References}

[1] H. Nishimori, Statistical Physics of Spin Glasses and Information Processing: An Introduction, Clarendon Press, Oxford, UK, 2001.

[2] T. Roska, "Computational and computer complexity of analogic cellular wave computers," in Proceedings of the 7th IEEE International Workshop on Cellular Neural Networks and Their Applications (CNNA '02), pp. 323-338, Frankfurt, Germany, July 2002.

[3] L. O. Chua and T. Roska, "The CNN paradigm," IEEE Transactions on Circuits and Systems I, vol. 40, no. 3, pp. 147156, 1993.

[4] L. O. Chua and L. Yang, "Cellular neural networks: theory," IEEE Transactions on Circuits and Systems, vol. 35, no. 10, pp. 1257-1272, 1988.

[5] T. Roska and L. O. Chua, "The CNN universal machine: an analogic array computer," IEEE Transactions on Circuits and Systems II, vol. 40, no. 3, pp. 163-173, 1993.

[6] A. Rodríguez-Vázquez, G. Liñán-Cembrano, L. Carranza, et al., "ACE16k: the third generation of mixed-signal SIMDCNN ACE chips toward VSoCs," IEEE Transactions on Circuits and Systems I, vol. 51, no. 5, pp. 851-863, 2004.

[7] Á. Zarándy and C. Rekeczky, "Bi-i: a standalone ultra high speed cellular vision system," IEEE Circuits and Systems Magazine, vol. 5, no. 2, pp. 36-45, 2005.

[8] http://www.anafocus.com.

[9] T. Roska, L. O. Chua, D. Wolf, T. Kozek, R. Tetzlaff, and F. Puffer, "Simulating nonlinear waves and partial differential equations via CNN-part I: basic techniques," IEEE Transactions on Circuits and Systems I, vol. 42, no. 10, pp. 807-815, 1995.

[10] T. Kozek, L. O. Chua, T. Roska, et al., "Simulating nonlinear waves and partial differential equations via $\mathrm{CNN}$ - part II: typical examples," IEEE Transactions on Circuits and Systems I, vol. 42, no. 10, pp. 816-820, 1995.

[11] J. M. Cruz and L. O. Chua, "Application of cellular neural networks to model population dynamics," IEEE Transactions on Circuits and Systems I, vol. 42, no. 10, pp. 715-720, 1995.

[12] K. R. Crounse, T. Yang, and L. O. Chua, "Pseudo-random sequence generation using the CNN universal machine with applications to cryptography," in Proceedings of the 4th IEEE International Workshop on Cellular Neural Networks and Their Applications (CNNA '96), pp. 433-438, Seville, Spain, June 1996. 
[13] K. R. Crounse and L. O. Chua, "Methods for image processing and pattern formation in Cellular Neural Networks: a tutorial," IEEE Transactions on Circuits and Systems I, vol. 42, no. 10, pp. 583-601, 1995.

[14] M. Ercsey-Ravasz, T. Roska, and Z. Néda, "Perspectives for Monte Carlo simulations on the CNN universal machine," International Journal of Modern Physics C, vol. 17, no. 6, pp. 909-922, 2006.

[15] M. Ercsey-Ravasz, T. Roska, and Z. Néda, "Stochastic simulations on the cellular wave computers," European Physical Journal B, vol. 51, no. 3, pp. 407-411, 2006.

[16] S. F. Edwards and P. W. Anderson, "Theory of spin glasses," Journal of Physics F, vol. 5, no. 5, pp. 965-974, 1975.

[17] D. Sherrington and S. Kirkpatrick, "Solvable model of a spinglass," Physical Review Letters, vol. 35, no. 26, pp. 1792-1796, 1975.

[18] F. Barahona, "On the computational complexity of Ising spin glass models," Journal of Physics A, vol. 15, no. 10, pp. 32413253, 1982.

[19] S. Istrail, "Statistical mechanics, three-dimensionality and NPcompleteness. I. Universality of intractability for the partition function of the Ising model across non-planar lattices," in Proceedings of the 32nd Annual ACM Symposium on Theory of Computing (STOC '00), pp. 87-96, Portland, Ore, USA, May 2000.

[20] M. Mezard, G. Parisi, and M. A. Virasoro, Spin Glass Theory and Beyond, World Scientific, Singapore, 1987.

[21] G. Rowe, Theoretical Models in Biology: The Origin of Life, the Immune System, and the Brain, Clarendon Press, Oxford, UK, 1997.

[22] R. N. Mantegna and H. E. Stanley, An Introduction to Econophysics: Correlations and Complexity in Finance, Cambridge University Press, Cambridge, UK, 2000.

[23] N. Sourlas, "Spin-glass models as error-correcting codes," Nature, vol. 339, no. 6227, pp. 693-695, 1989.

[24] S. Kirkpatrick, C. D. Gelatt Jr., and M. P. Vecchi, "Optimization by simulated annealing," Science, vol. 220, no. 4598, pp. 671-680, 1983.

[25] T. Roska and Á. Rodríguez-Vázquez, "Toward visual microprocessors," Proceedings of the IEEE, vol. 90, no. 7, pp. 1244 1257, 2002. 